\title{
The Role of Whole-blood Parameters in Predicting the Severity of Acute Rheumatic Carditis in Children
}

\author{
- Yeter Duzenli Kar, ๑ Ufuk Utku Gullu* \\ Afyonkarahisar Health Sciences University Faculty of Medicine, Department of Pediatrics, Division of Pediatric Hematology and Oncology, \\ Afyonkarahisar, Turkey \\ Erzurum Regional Training and Research Hospital, Clinic of Pediatrics, Division of Pediatric Hematology and Oncology, Erzurum, Turkey \\ *Erzurum Regional Training and Research Hospital, Clinic of Pediatrics, Division of Pediatric Cardiology, Erzurum, Turkey
}

\section{Abstract}

\begin{abstract}
Aim: Acute rheumatic fever (ARF) is the most common cause of acquired heart disease especially in developing countries. This study is intended to evaluate the relationship between severity of cardiac involvement and hematological parameters in children diagnosed with carditis at the first episode of ARF.
\end{abstract}

Methods: In this study, 45 children with carditis at the first episode of ARF and 30 healthy children of the same age and gender, between September 2015 and February 2019, were retrospectively reviewed. The data of the patients were recorded from the hospital automation system. Patients with acute rheumatic carditis (ARC) were grouped into two as mild ( $\mathrm{n}=30)$ and moderate-severe ARC $(n=15)$. Hemoglobin, red blood cell distribution width (RDW), leukocyte, neutrophil, monocyte, lymphocyte, platelet counts, neutrophil-to-lymphocyte (NLR), platelet-to-lymphocyte (PLR), monocyte-to-lymphocyte ratio (MLR) were compared between control and all carditis patients, mild and moderate-severe carditis.

Results: In ARC patients, hemoglobin and RDW values were statistically significantly lower ( $<<0.0001, p=0.0001$, respectively) and leukocyte, neutrophil, platelet, NLR, PLR, MLR were statistically significantly higher $(p<0.0001, p<0.0001, p=0.0001, p<0.0001$, $p=0.0022, p=0.0032$, respectively) than in control. A statistically significant difference was determined between hemoglobin, RDW in mild and moderate-severe carditis $(p<0.05)$.

Conclusions: In our study, it was found that RDW increased significantly in moderate-severe carditis compared to mild ARC and could be used as an indicator of severe carditis in ARF.

Keywords: Child, red blood cell distribution width, rheumatic fever, rheumatic heart disease

\section{Introduction}

Acute rheumatic fever (ARF) is a systemic, inflammatory disease which occurs with the group a beta-hemolytic streptococcus emerges 1-5 weeks after upper respiratory tract infection and it can affect the joints, brain, heart, blood vessels, skin and subcutaneous connective tissue (1). It is seen especially in developing countries and it is the most common cause of acquired heart disease in children and young adults in many parts of the world $(1,2)$. Around 500,000 new cases of ARF are seen annually worldwide, and about 15 million people are known to have chronic rheumatic heart disease (2). Carditis is detected in $50-80 \%$ of ARF patients and most commonly affects the mitral and aortic valves (1-3). Carditis is the most common and major symptom after arthritis that can lead to acute heart failure and chronic valve disease and increase mortality. Although the endocardium is frequently affected, myocardium and pericardium may also rarely be affected $(2,4)$. Severe carditis occurs at the rate of $20 \%$ approximately and may cause congestive heart failure (2).

Blood cell interactions play an important role in inflammation, immune response, oncogenesis and

Yazışma Adresi/Address for Correspondence: Yeter Duzenli Kar, Afyonkarahisar Health

Sciences University Faculty of Medicine, Department of Pediatrics, Division of Pediatric Hematology and Oncology, Afyonkarahisar, Turkey

Phone: +90 5553162650 E-mail: yeterduzenli@yahoo.com ORCID: orcid.org/0000-0003-2917-7750

Received: 30.12.2020 Accepted: 06.02.2021

Copyright 2021 by The Medical Bulletin of istanbul Haseki Training and Research Hospital The Medical Bulletin of Haseki published by Galenos Yayinevi. 
hemostasis. It is known that neutrophils are indicative of nonspecific inflammation, lymphocytes are involved in immune response regulation and platelets play an important role in inflammation by secreting various chemokines and cytokines (5). The relationship between acute rheumatic carditis (ARC) and systemic inflammation was previously studied in several studies, but there are a limited number of studies on children (5-7).

The relationship between inflammation markers such as neutrophil-to-lymphocyte ratio (NLR), plateletto-lymphocyte ratio (PLR), monocyte-to-lymphocyte ratio (MLR), red blood cell distribution width (RDW), platelet count, easily calculated from whole blood count parameters, and the severity of many inflammatory diseases such as cancer, systemic lupus erythematosus, psoriasis, atopic dermatitis, Kawasaki, ARF was investigated (5-10).

The aim of the study was to examine the relationship between whole blood parameters in children with cardiac involvement at the time of ARF diagnosis (hemoglobin, RDW, mean corpuscular volume (MCV), leukocyte, neutrophil, monocyte, lymphocyte and platelet counts) and hematological indices derived from these whole blood parameters NLR, PLR, MLR with carditis severity.

\section{Methods}

\section{Study Design}

This study was approved by the local ethics committee (07.10.2019, 2019/12-121) (number: 37732058/51410). and written informed consent was obtained from the parents or guardians of both the patients and the controls. Between September 2015 and February 2019, 45 children diagnosed with ARC at the first episode of ARF at our hospital Pediatric Cardiology Clinic and 30 healthy children of the same age and gender were included in the study. There were no pre-existing rheumatic illnesses. Patients with a history of nonsteroid anti-inflammatory drug, corticosteroid use within 4 days before applying to hospital, patients with impaired renal and kidney function, patients with thalassemia carriage and hemolytic anemia, those with iron, folate and B12 deficiency anemia, patients with signs of infection, patients with reproduction in blood and urine cultures, and patients with a history of antibiotic use in the past week were excluded from the study. Healthy control group was selected from healthy volunteers who were referred to our hospital for cardiac murmur, who underwent electrocardiogram and echocardiography, and had no history or symptoms of acute or chronic disease. Physical examination of all patients and healthy control group was performed by the same pediatric cardiologist.

ARF was diagnosed using modified Jones criteria and by the differential diagnosis of all other causes (11).

\section{Echocardiographic Assessment}

Echocardiographic examinations of all patients were performed by an experienced pediatric cardiologist before initiating anti-inflammatory treatment. The Vivid 5 Pro Ultrasound System (GE MedicalSystems, NE) was used for two-dimensional, M-modeandcolour-flow Doppler imaging. For the echocardiographic diagnosis of pathological valve insufficiency, the criteria previously defined and then updated by the World Heart Federation in 2012 were used. For mitral valve insufficiency, the following should be present: insufficiency jet should be seen at least two different sections, jet length should be at least $2 \mathrm{~cm}$, and during systole peak flow rate should be at least $3 \mathrm{~m} / \mathrm{s}$. For aortic valve insufficiency were as follows: insufficiency jet should be seen in at least two different sections, jet length should be at least $1 \mathrm{~cm}$, and peak flow velocity along the diastole should be $>3 \mathrm{~m} / \mathrm{s}$ (12). Mitral and aortic regurgitation were classified as mild, moderate, or severe according to published standards (13). For the echocardiographic diagnosis of mild-moderate-severe carditis, the criteria previously defined were used (14).

\section{Mild Carditis}

(Valvular regurgitation is relatively mild in the absence of pre-existing disease; in first episodes of ARF)

Mild mitral or aortic regurgitation clinically and/or on echo, with no evidence of cardiac chamber enlargement on chest X-ray, electrocardiography, or echo and no clinical evidence of heart failure.

\section{Moderate Carditis}

Any valve lesion of moderate severity clinically (e.g., mild or moderate cardiomegaly), or echocardiographic demonstration of enlargement in any of the heart chambers or any moderate severity valve lesion on echo: (When there is both mitral and aortic regurgitation, one must be moderate by echo criteria for the carditis to be classified of moderate severity), mitral regurgitation is considered moderate if there is a broad high-intensity proximal jet filling half the left atrium or a lesser volume high-intensity jet producing prominent blunting of pulmonary venous inflow. Aortic regurgitation is considered moderate if the diameter of the regurgitant jet is $15 \%-30 \%$ of the diameter of the left ventricular outflow tract with flow reversal in the upper descending aorta.

\section{Severe Carditis}

Any impending or previous cardiac surgery for rheumatic heart disease, or any severe valve lesion (significant cardiomegaly, and/or heart failure), or any severe valve lesion on echo: Doppler flow patterns and abnormal regurgitant color in pulmonary veins are a prerequisite for severe mitral regurgitation. Reversing 
in lower descending aorta is required for severe aortic regurgitation.

\section{Blood Samples}

Patient data were retrospectively reviewed. The full blood count analysis was done by Sysmex XN-10 analyzer in the laboratory of our institution. Hemoglobin, MCV, RDW, leukocyte count, neutrophil count, monocyte count, lymphocyte count, platelet count, NLR, PLR, $M L R$, erythrocyte sedimentation rate, C-reactive protein levels (CRP), anti-streptolysin-O (ASO) levels at the time of diagnosis were retrieved from the patient charts. The NLR was calculated as a simple ratio between absolute neutrophil and absolute lymphocyte counts, the PLR was calculated as a simple ratio between platelet and absolute lymphocyte counts, the MLR was calculated as a simple ratio between absolute monocytel and absolute lymphocyte counts.

\section{Statistical Analysis}

Statistical Packageforthe Social Sciences 15.0 version statistical packet program was used for statistical analysis of the data. Descriptive statistics were expressed as number and percentage for categorical variables, and as mean and standard deviation for numerical variables. In the analyses comparing the groups, Mann-Whitney $U$ test was used for continuous variables in comparison of two groups and chi-square test and Fisher's Exact test were used for categorical variables. Results were evaluated at $95 \%$ confidence interval and significance was evaluated at $p<0.05$ level.

\section{Results}

Group a total of 45 ARC patients and 30 healthy children were included in the study. The mean age of the patient group ARC was 11.87 \pm 2.47 (range=7.5-17.1) years and the female/male ratio was $27 / 18$. The mean age of the control group was $11.53 \pm 2.75$ (range $=7-17$ ) years and the female/male ratio was 15/15. In ARC patients, hemoglobin, RDW values were statistically significantly lower ( $p<0.0001, p=0.0001$, respectively) and leukocyte, neutrophil, platelet counts and NLR, PLR, MLR ratio were significantly higher than healthy control group $(p<0.0001$, $p<0.0001, p=0.0001, p<0.0001, p=0.0022, p=0.0032$ ， respectively) (Table 1). The hemoglobin, MCV, RDW, leukocyte, neutrophil, monocyte, lymphocyte, and platelet counts and the NLR, PLR, MLR, erythrocyte sedimentation rate, CRP and ASO levels values of the patient and control groups were shown in Table 1.

The Distribution of Heart Valve Involvement in Patients Diagnosed with ARC and the Relationship Between Hematological Parameters and Severity of Cardiac Involvement

Two of our patients diagnosed with ARC had aortic regurgitation, 19 patients had mitral insufficiency and 24 of them aortic regurgitation and mitral insufficiency.

Of 45 patients with ARC, 30 had mild, 10 had moderate, and five had severe ARC. The mean age of 30 patients with mild ARC was $12.02 \pm 2.49$ years, and the female/male ratio was $15 / 15$; the mean age of 15 patients with moderate-severe ARC was $11.58 \pm 2.48$

\begin{tabular}{|c|c|c|c|}
\hline Characteristics & Control group & Acute rheumatic carditis & $p$ \\
\hline Age (year) (mean \pm SD) & $11.53 \pm 2.75$ & $11.87 \pm 2.47$ & $0.5805^{a}$ \\
\hline Sex (Female/Male) (n (\%)) & $15 / 15(50 \% / 50 \%)$ & $27 / 18(60 \% / 40 \%)$ & $0.3927^{b}$ \\
\hline $\mathrm{Hb}(\mathrm{g} / \mathrm{dL})($ mean $\pm \mathrm{SD})$ & $14.29 \pm 0.91$ & $12.38 \pm 1.57$ & $<0.0001^{a}$ \\
\hline $\mathrm{MCV}(\mathrm{fL})($ mean $\pm \mathrm{SD})$ & $82.08 \pm 4.43$ & $80.45 \pm 5.36$ & $0.3223^{a}$ \\
\hline $\mathrm{RDW}(\%)($ mean $\pm \mathrm{SD})$ & $13.15 \pm 0.70$ & $12.05 \pm 1.44$ & $0.0001^{\text {a }}$ \\
\hline Leukocyte $\left(/ \mathrm{mm}^{3}\right)($ mean $\pm \mathrm{SD})$ & $6637.67 \pm 1439.83$ & $11555.51 \pm 2847.88$ & $<0.0001^{a}$ \\
\hline Neutrophil $\left(/ \mathrm{mm}^{3}\right)($ mean $\pm \mathrm{SD})$ & $3300.67 \pm 1183.27$ & $7942.40 \pm 2698.86$ & $<0.0001^{a}$ \\
\hline Lymphocyte $\left(/ \mathrm{mm}^{3}\right)($ mean $\pm \mathrm{SD})$ & $2436.33 \pm 595.32$ & $2534.00 \pm 886.29$ & $0.8967^{a}$ \\
\hline Platelet $\left(/ \mathrm{mm}^{3}\right)$ (mean $\left.\pm \mathrm{SD}\right)$ & $317566.67 \pm 75275.90$ & $435812.22 \pm 136986.25$ & $0.0001^{a}$ \\
\hline NLR (mean $\pm \mathrm{SD})$ & $1.45 \pm 0.70$ & $4.01 \pm 4.25$ & $<0.0001^{a}$ \\
\hline $\mathrm{PLR}($ mean $\pm \mathrm{SD})$ & $137.93 \pm 46.10$ & $207.07 \pm 194.14$ & $0.0022^{a}$ \\
\hline $\operatorname{MLR}($ mean $\pm S D)$ & $0.24 \pm 0.10$ & $0.42 \pm 0.51$ & $0.0032^{a}$ \\
\hline ASO $(U / m L)($ mean $\pm S D)$ & - & $1057.24 \pm 541.81$ & - \\
\hline ESR (mm/hour) (mean $\pm \mathrm{SD})$ & - & $41.22 \pm 18.05$ & - \\
\hline $\mathrm{CRP}(\mathrm{mg} / \mathrm{L})($ mean $\pm \mathrm{SD})$ & - & $7.39 \pm 6.23$ & - \\
\hline \multicolumn{4}{|c|}{ 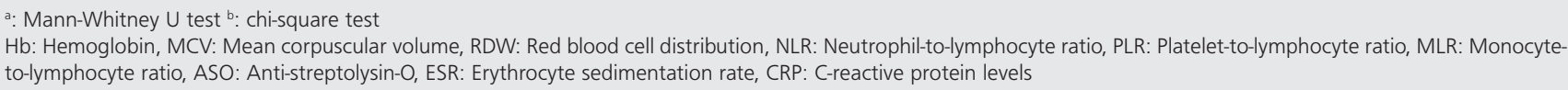 } \\
\hline
\end{tabular}


years and the female/male ratio was 12/3. A significant difference was found in hemoglobin, MCV, RDW between mild and moderate-severe ARC $(p<0.05)$. The duration of hospitalization was significantly longer in moderatesevere ARC patients than in mild ARC patients $(p<0.01)$. Erythrocyte sedimentation rate, CRP and ASO levels values were higher in moderate-severe ARC patients compared to mild ARC patients (Table 2).

\section{Discussion}

In our study, two-thirds of the patients diagnosed with ARC at the first episode of ARF were mild carditis cases. We found that severe ARC cases were less frequent in accordance with the literature $(2,14)$. Today, we think that there are fewer cases of severe ARC because of better living conditions, easier access to health care services, early diagnosis of the disease, adequate and timely initiation of penicillin treatment and prophylaxis, better patient followup and the application of improved diagnostic methods such as echocardiography in almost all centers.

Although the pathogenesis of the disease is not fully elucidated, the most accepted theory is crossreactivity. Accordingly, depending on the similarity of some streptococcal antigens to tissue antigens, it can be explained that immunological reactions resulting from activation of the humoral and cellular immune system cause inflammatory response (3). In the acute phase of ARF, disruption of connective tissue and edema occur due to infiltration of myocardium and valve by T-lymphocytes,
B-lymphocytes, macrophages and mast cells. In the healing process of ARC, various degrees of fibrosis and valve damage are seen in the heart (7). Although any layer of the heart may be affected in cardiac involvement, endocardial and myocardial involvement is the most common. As a serious complication of the disease, congestive heart failure, which is indicative of myocarditis, may develop and the disease may be fatal. Valve lesions occur due to endocardial involvement. Mitral and aortic valves are affected most commonly $(2,3)$. In our study, congestive heart failure secondary to myocarditis was not detected in our patients. In accordance with the literature, mitral and aortic valves were the most commonly affected valves.

In recent years, there are many studies investigating neutrophil, lymphocyte and platelet count, NLR, mean platelet volume values and cardiovascular disease severity. In a study in adults, Akboga et al. (15) found that RDW value was higher in patients with chronic rheumatic mitral valve stenosis as an indicator of persistent chronic inflammation than in the healthy control group. In the same study, no statistically significant difference was found in NLR in patients with chronic rheumatic mitral valve stenosis compared to the healthy control group, but they found a positive correlation between RDW and NLR/CRP. They suggested that RDW is an independent predictor risk factor for chronic rheumatic mitral valve stenosis. Ozturk et al. (16) reported that NLR elevation could be used as an independent predictor of spontaneous echocardiographic

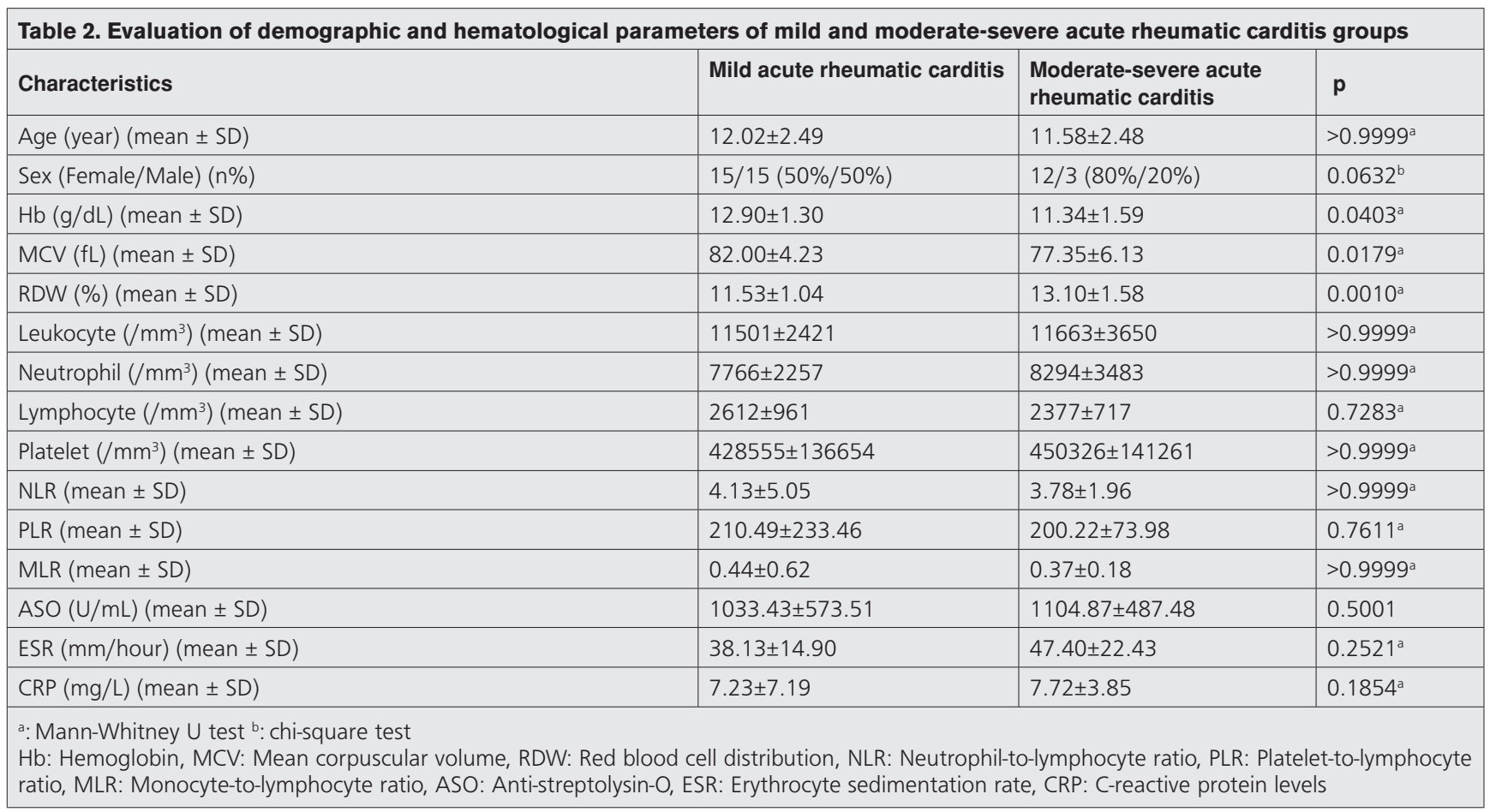


contrast in patients with rheumatic mitral stenosis. Polat et al. (17) reported that as the severity of rheumatic mitral valve stenosis increases, NLR increases as well, and when the NLR cut off value is taken as 2.56, it has the power to predict severe rheumatic mitral valve stenosis with 75\% sensitivity and $74 \%$ specificity. They reported that NLR is useful in predicting both the presence and severity of rheumatic mitral valve stenosis. Ozdemir et al. (18), in accordance with our study, stated that hemoglobin value was significantly lower but leukocyte and platelet value were significantly higher in ARC patients compared to the control group; Küçük et al. (19), however, reported that leucocyte and platelet count in ARC patients was statistically significantly higher than in the control group. They also hypothesized that high levels of RDW after ARC treatment may be used as an indicator of heart valve stenosis in the later years. Similar to the results of our study, Asık et al. (20) reported that neutrophil, leukocyte, NLR were significantly increased in patients with ARF compared to healthy children, and these parameters could be used in the diagnosis of ARF. Karpuz et al. (21) reported that RDW in children with ARF was significantly higher than healthy controls. They suggested that in the chronic phase of ARF, RDW showed a positive correlation with CRP and erythrocyte sedimentation rate, which are chronic inflammation markers, and that RDW could be used as a diagnostic marker in the diagnosis of the disease. In our study, similar to the study by Çelik et al. (7) on child cases consisting of those with ARC and healthy control group, we found that hemoglobin and RDW values were lower and leukocyte, neutrophil, platelet count and NLR were higher in ARC. In addition, in our study, hemoglobin and MCV values were significantly lower in moderatesevere ARC patients compared to mild ARC patients and RDW values were significantly higher in moderate-severe carditis patients. We can infer that hemoglobin levels of patients reduce when carditis severity increases because the patients are susceptible to inflammation anemia due to increased carditis severity. Unlike the literature, we think that the reason why we did not find any relationship between ARC patients and NLR in our study was because we included $A R C$ patients at the first episode, so patients were accompanied by one or more of the modified Jones Criteria and other major or minor criteria for the diagnosis of ARF in addition to carditis. In addition, we think that it is because there are adult studies in the literature and therefore the relationship between the degree of mitral stenosis and hematological indices parameters has been examined.

\section{Study Limitations}

The limitations of our study were the low number of patients and the presentation of a single-center experience.
The fact that it is a retrospective study, it can't evaluate post-treatment measurements and the absence of longterm follow-up are the limitations of our study. However, our study is important because it is one of the rare studies on the subject in children with ARC.

\section{Conclusion}

There are a limited number of studies examining the relationship between the degree of cardiac involvement and hematological parameters in patients diagnosed with ARC at the first episode of ARF in children. In our study, it was shown that RDW increased significantly in moderatesevere ARC patients compared to mild ARC patients and RDW could be used as an indicator of severe carditis in ARF. However, since the severity of inflammation may change due to the presence of different major and minor criteria for the diagnosis of ARF in ARC patients, it was detected that leukocyte, neutrophil, platelet, monocyte, $N L R, P L R, M L R$ levels were not guiding in predicting the degree of carditis in ARC.

\section{Authorship Contributions}

Concept: Y.D.K., U.U.G., Design: Y.D.K., Data Collection or Processing: Y.D.K., U.U.G., Analysis or Interpretation: Y.D.K., U.U.G., Literature Search: Y.D.K., U.U.G., Writing: Y.D.K.

Conflict of Interest: The authors declared no conflicts of interest concerning the authorship and publication of this article.

Financial Disclosure: The authors received no financial support for the research and/or authorship of this article

\section{References}

1. Marijon E, Mirabel M, Celermajer DS, Jouven X. Rheumatic heart disease. Lancet 2012;379:953-64.

2. Webb RH, Grant C, Harnden A. Acute rheumatic fever. BMJ 2015;351:3443.

3. Carapetis JR, Beaton A, Cunningham MW, et al. Acute rheumatic fever and rheumatic heart disease. Nat Rev Dis Primers 2016;2:15084.

4. Eroğlu AG. Update on diagnosis of acute rheumatic fever: 2015 Jones criteria. Turk Pediatri Ars 2016;51:1-7.

5. Gasparyan AY, Ayvazyan L, Mukanova U, Yessirkepov M, Kitas GD. The Platelet-to-Lymphocyte Ratio as an Inflammatory Marker in Rheumatic Diseases. Ann Lab Med 2019;39:34557.

6. Akboğa MK, Akyel A, Şahinarslan A, et al. Neutrophil-tolymphocyte ratio is increased in patients with rheumatic mitral valve stenosis? Anatol J Cardiol 2015;15:380-4.

7. Çelik SF, Çelik E. The neutrophil-to-lymphocyte ratio and mean platelet volume can be associated with severity of valvular involvement in patients with acute rheumatic carditis. Cardiovasc J Afr 2018;29:296-300. 
8. Jiang $Y, M a ~ W$. Assessment of Neutrophil-to-Lymphocyte Ratio and Platelet-to-Lymphocyte Ratio in Atopic Dermatitis Patients. Med Sci Monit 2017;23:1340-6.

9. Demir F, Karadeniz C, Özdemir R, et al. Usefulness of Neutrophil to Lymphocyte Ratio in Prediction of Coronary Artery Lesions in Patients with Kawasaki Disease. Balkan Med J 2015;32:371-6.

10. Yin $X$, Xiao $Y$, Li F, Qi S, Yin Z, Gao J. Prognostic Role of Neutrophil-to-Lymphocyte Ratio in Prostate Cancer: A Systematic Review and Meta-analysis. Medicine (Baltimore) 2016;95:2544.

11. Gewitz MH, Baltimore RS, Tani LY, et al. Revision of the Jones Criteria for the Diagnosis of Acute Rheumatic Fever in the Era of Doppler echocardiography: A Scientific Statement from the American Heart Association. Circulation 2015;131:180618.

12. Reményi B, Wilson N, Steer A, et al. World Heart Federation criteria for echocardiographic diagnosis of rheumatic heart disease-an evidence-based guideline. Nat Rev Cardiol 2012;9:297-309

13. Kayali S, Belder N. Subclinical rheumatic heart disease: $A$ single center experience. North Clin Istanb 2018;5:329-33.

14. Atatoa-Carr P, Lennon D, Wilson N. New Zealand Rheumatic Fever Guidelines Writing Group. Rheumatic fever diagnosis, management, and secondary prevention: a New Zealand guideline. N Z Med J 2008;121:59-69.

15. Akboğa MK, Abacı A, Canpolat U, et al. Kırmızı kan hücresi dağılım genişliğinin romatizmal mitral kapak darlığının varlığı ve ciddiyetiyle ilişkisi [Association of red blood cell distribution width with presence and severity of rheumatic mitral valve stenosis]. Turk Kardiyol Dern Ars 2015;43:227-33.

16. Öztürk D, Erturk M, Celık O, et al. The role of the neutrophil/ lymphocyte ratio in patients with rheumatic mitral stenosis as an indicator of spontaneous echocardiographic contrast. Kardiologia Polska (Polish Heart J) 2014;72(10):969-76.

17. Polat N, Yildiz A, Yuksel M, et al. Association of neutrophillymphocyte ratio with the presence and severity of rheumatic mitral valve stenosis. Clin Appl Thromb Hemost 2014;20:7938.

18. Ozdemir R, Karadeniz C, Doksoz O, et al. Are mean platelet volume and platelet distribution width useful parameters in children with acute rheumatic carditis? Pediatr Cardiol 2014;35:53-6.

19. Kucuk M, Ozdemir R, Karadeniz C, et al. Red blood cell distribution width: can it be a predictive marker for longterm valvular involvement in children with acute rheumatic carditis? Int J Lab Hematol 2016;38:569-75.

20. Asik A, Duru NS, Elevli M. An Evaluation of Platelet Parameters and Neutrophil/Lymphocyte Ratios in Children with Acute Rheumatic Fever. The Journal of Pediatric Research 2019;6:3744.

21. Karpuz D, Giray D, Ozyurt A, Bozlu G, Unal S, Hallioglu O. Can whole-blood parameters be used in follow-up of children with rheumatic valvular heart disease? Cardiol Young 2017; 27:764-9. 